\title{
The Biotic Pump: \\ Condensation, atmospheric dynamics and climate
}

\author{
Anastassia M. Makarieva* \\ and Victor G. Gorshkov
}

Theoretical Physics Division,

Petersburg Nuclear Physics Institute,

Gatchina, 188300, St. Petersburg, Russia

E-mail: elba@peterlink.ru E-mail: vigorshk@thd.pnpi.spb.ru

${ }^{*}$ Corresponding author

\begin{abstract}
Intense condensation associated with high evaporation from natural forest cover maintains regions of low atmospheric pressure on land. This causes moist air to flow from ocean to land, which compensates the river runoff. Deforestation induces large-scale desiccation by disrupting this flow. Here we overview this theory and quantify the horizontal pressure gradients that govern the continental moisture supply. High evaporation and extensive natural forests guarantee both a stable and high throughput hydrological cycle. Forests protect a continent against devastating floods, droughts, hurricanes, and tornadoes. Sustaining natural forests is a sound strategy for water security and climate stabilisation.
\end{abstract}

Keywords: biotic pump; deforestation; condensation; evaporation; transpiration; leaf area; atmospheric circulation; hydrological cycle; climate; desertification.

Reference to this paper should be made as follows: Makarieva, A.M. and Gorshkov, V.G. (2010) 'The Biotic Pump: Condensation, atmospheric dynamics, and climate', Int. J. Water, Vol. 5, No. 4, pp.365-385.

Biographical notes: Anastassia M. Makarieva has degrees in Biophysics and Atmospheric Science and is currently Senior Scientific Researcher in the Theoretical Physics Division of the Petersburg Nuclear Physics Institute, Russia. She is a member of the International Expert Group on Earth System Preservation and a recipient of the L'Oreal-UNESCO-Russian Academy of Sciences Prize. With Victor Gorshkov, she pioneered the theory of the biotic pump, which challenges standard assumptions in atmospheric physics: 'Biotic pump of atmospheric moisture as driver of the hydrological cycle on land', Hydrology and Earth System Sciences (2007).

Victor G. Gorshkov is a Theoretical Physicist at the Petersburg Nuclear Physics Institute, Russia. He has advised on many environmental projects in Russia, the Scientific Committee of the International Geosphere Biosphere Program, the UNESCO Encyclopedia of Life Support Systems, the Global Environment Facility, and the Editorial Board of Ecological Complexity. He is also a member of the International Expert Group on Earth System Preservation. $\mathrm{He}$ is the author of Physical and Biological Bases of Life Stability: Man, Biota, Environment (1995) and co-author with Makarieva of Biotic Regulation of the Environment: Key Issue of Global Change (2000). 


\section{Introduction}

\subsection{The transient character of fresh water stocks on land}

Life on land is only possible when the soil contains enough moisture for green plants to grow. As land is elevated above the sea, the fresh water accumulated on land runs down to the ocean along the continental slope and leaves land as river runoff. The global renewable stock of fresh water on land is very small: all the water contained in glaciers, lakes and soil could be depleted by global river runoff in just a few years (L'vovitch, 1979).

In the presence of such a significant leak, the stock of fresh water on land will remain stable only if there is an equally intense reverse transport of fresh water from ocean to land. River runoff is characterised by high power: this is clear from the fact that electric hydropower contributes considerably to the energy budget of our civilisation (Makarieva et al., 2008). Likewise, the reverse ocean-to-land moisture transport demands significant energy inputs. Transport of liquid water would involve large losses owing to land surface friction. The only physically realistic mechanism of delivering moisture to land is by means of the atmosphere, making use of the gaseous phase of water. Thus, the continuous river runoff of liquid water from land to ocean is compensated by moist winds that transport gaseous water in the opposite direction. Water vapour carried from the ocean by winds condenses and precipitates over land.

When evaluating water security strategies, one has to bear in mind the transient nature of the fresh water stock on land. By way of comparison, global food stocks are also short: the global grain stock corresponds to just a few months of global consumption (Holt-Giménez and Kenfield, 2008). This means that if the global photosynthesising mechanism of green crops degrades, famine will ensue worldwide in just a few months. Similarly, if the ocean-to-land transport of moisture stalls, the fresh water will totally disappear in a few years. Just as global food security is based on the persistent biological productivity of green plants, so global and regional climate patterns and water security critically depend on the integrity of mechanisms that transport atmospheric moisture from ocean to land.

\subsection{Ecological mechanism of ocean-to-land moisture transport: a biotic pump}

In this paper, we review accumulated evidence (Makarieva and Gorshkov, 2007, 2009a, 2009b; Makarieva et al., 2009; Sheil and Murdiyarso, 2009), as well as present new considerations showing that the delivery of moisture to land is a function of the state of terrestrial vegetation cover. The process of evolution solved two difficult problems - how to draw atmospheric moisture deep inland and how to make this moisture supply stable. Because of a high leaf area index, natural forests maintain high evaporation fluxes (transpiration and interception), which can exceed evaporation fluxes over the oceanic surface. Evaporation and transpiration cause an elevated moisture content in the vertical column of air over a forest, which, in turn, produces elevated condensation well above the canopy owing to the adiabatic ascent of moist air. The loss of gas out of the air column from condensation decreases air pressure well above the canopy, causing an increased vertical pressure gradient that results in an ascending air 
motion over the forest. This 'sucks in' air horizontally from elsewhere via the formation of horizontal pressure gradients, and under the right circumstances, can bring moist air from the ocean. Moist ocean air enhances condensation above the forest, further strengthening the process and thus stabilising land moisture supply. Air returns to the ocean in the upper atmosphere after precipitation of moisture over the continent, completing the circulation pattern. Such biotically induced atmospheric circulation sustaining the hydrological cycle on land has been termed the 'biotic pump' of atmospheric moisture (Makarieva and Gorshkov, 2007). However, following human deforestation practices, this cycle of moisture transport stalls, Figure 1.

Figure 1 The impact of vegetation on the hydrological cycle and atmospheric circulation: (left) natural biotic pump and (right) cleared vegetation. Forest evaporation (green arrow) enhances condensation, lowering pressure and inducing ocean-to-land moist air flows (blue arrow) penetrating deep inland. Image by Douglas Sheil

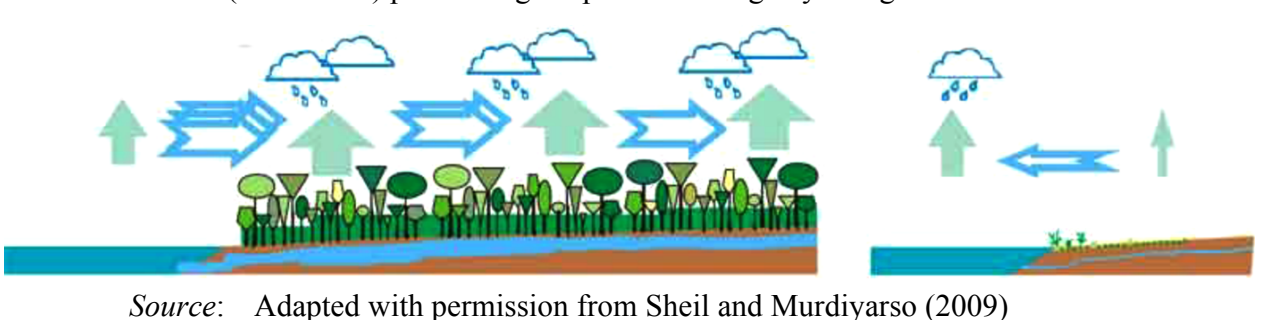

Source: Adapted with permission from Sheil and Murdiyarso (2009)

Remarkably, these effects of condensation/evaporation on air pressure through the removal or addition of vapour molecules have been overlooked by the international scientific community. As a result, there is confusion among many scientists over whether condensation increases or reduces moist air pressure (Makarieva et al., 2010). A lack of theoretical concepts for the analysis of moist atmosphere is a major obstacle for the development of atmospheric circulation theory (Schneider, 2006). Thus, while a variety of impacts of regional vegetation cover have been investigated, including changes in albedo and re-distribution of latent and sensible heat fluxes (Pielke, 2001; National Research Council, 2005; Bonan, 2008), the potential role of forests as generators of pressure gradients driving moist ocean-to-land winds has not been subjected to a wide scrutiny.

The paper is structured as follows. In Section 2, we outline the principles of condensation-induced atmospheric dynamics, and the physical mechanism of the biotic pump. In Section 3, we derive the horizontal pressure gradients induced by water vapour condensation for the general case of a non-isothermal surface. In Section 4, the notions of donor and acceptor regions are introduced, and the spatial structure of condensation-induced circulation in the presence of a horizontal temperature gradient is discussed. In Section 5, we investigate the role of evaporation in maintaining these pressure gradients on an isothermal surface. In Section 6, the problem of stabilising the flow of moist air by an extensive forest cover is discussed, and the size of a territory that has to be covered by natural forests to ensure a stable moisture supply from ocean to land is estimated. In Section 7, the difference in functioning of natural forests and non-forest vegetation is illustrated. 


\section{Basic physical principles of condensation-induced atmospheric dynamics}

Atmospheric air has the remarkable physical property of an ideal gas: air pressure does not depend on molar masses of air components. It is a function of total molar density and temperature. That is, at a fixed temperature, a given pressure can be obtained for a mixture of gases with arbitrary molar masses by setting the value of their total molar density. A second important property of atmospheric air is the presence of a condensable component - water vapour. The molar density of moist air is equal to the sum of molar densities of dry air components and water vapour. When the water vapour condenses, its molar density diminishes and the air pressure drops.

The physical cause of condensation is not trivial. Consider a motionless atmosphere with no greenhouse substances. Air temperature in such an atmosphere is the same at all heights. The partial pressures of all gases follow the hydrostatic Boltzmann distribution as determined by their molar masses. The Boltzmann distribution corresponds to an exponential decline of gas pressure with height above the planetary surface: the pressure drops $e$-fold (by 2.71 times) as the height increases by $h$. The value of $h$, the exponential scale height, has the meaning of the height of a uniformly dense atmosphere with the same total amount of gas: the heavier the gas, the more compressed its vertical distribution and the smaller its exponential scale height $h$. The exponential scale height of water vapour with molar mass $18 \mathrm{~g} \mathrm{~mol}^{-1}$ in such an atmosphere would be about $13 \mathrm{~km}$. In comparison, the major air components, nitrogen and oxygen, with similar molar masses $\sim 30 \mathrm{~g} \mathrm{~mol}^{-1}$, would have scale heights of approximately $8 \mathrm{~km}$. Water vapour would be saturated at the Earth's surface only (due to the contact with the liquid hydrosphere) and have an unsaturated pressure everywhere else in the atmospheric column. There would be no evaporation or condensation.

However, such a hypothetical static distribution of vertically isothermal moist air is unstable. Any random upward displacement of an air volume leads to adiabatic cooling of the rising air. Air temperature drops such that the equilibrium partial pressure of water vapour dictated by the Boltzmann distribution becomes oversaturated at all heights where the moist saturated air ascends. This causes the water vapour to condense. Its partial pressure decreases down to the saturated pressure. Condensation diminishes the total air pressure and disturbs the hydrostatic distribution of moist air. The vertical gradient of air pressure becomes greater than the weight of a unit air volume. There appears an upward directed force acting on a unit air volume. A static equilibrium of moist air in the gravitational field is not possible. The air begins to ascend along the non-equilibrium pressure gradient produced by condensation. Condensation is sustained by continuous evaporation of water vapour from the hydrosphere. The upward-directed force that acts on moist air and causes it to rise adiabatically is termed the evaporative-condensational force (Makarieva and Gorshkov, 2007, 2009a).

Importantly, the evaporative-condensational force causes air to ascend adiabatically, i.e., with no exchange of heat, over a large area with linear size exceeding the vertical scale height $h$ even if the atmosphere is initially vertically isothermal. This is not a buoyancy-related force traditionally invoked to explain convective instability. In a vertically isothermal atmosphere, a small air parcel with linear dimensions smaller than $h$ may occasionally ascend due to the Archimedes force - if temperature fluctuations make the parcel warmer than the surrounding air. But, as the air parcel cools while ascending, its positive buoyancy disappears and the motion extinguishes. 
Moreover, the mixing of such parcels with surrounding air destroys the adiabatic character of their motion.

Most condensed water vapour leaves the atmosphere as precipitation. A minor part is maintained in the atmosphere by the rising air flow. This imposes a drag force on rising air and reduces its vertical velocity. In Section 6, we discuss the significance of this gravitational drag for condensation-induced air motions. Unlike the flow of water vapour, which condenses while rising, the flow of dry air components, which conserve their mass, cannot be unidirectional (vertical), because the dry air does not disappear as it ascends. For this reason, regions of horizontal air flow will appear. Condensation of water vapour in the ascending air produces both vertical and horizontal pressure gradients. The presence of water vapour in the atmosphere contacting with a liquid hydrosphere leads to the formation of three-dimensional circulation patterns. To this point in time, these complex condensation-induced air motions have not received consideration from meteorologists.

\section{Horizontal pressure gradients induced by condensation}

The equations of state for moist air as a whole, as well as for its components - dry air and water vapour - include one and the same universal molar gas constant $R$ and do not depend on molar masses and mass densities of the components:

$$
p=N R T, p_{v}=N_{v} R T, p_{d}=N_{d} R T,
$$

where $p, N, p_{v}, N_{v}, p_{d}$ and $N_{d}$ are the pressure and molar density of moist air as a whole, water vapour and dry air, respectively, and $T$ is the absolute temperature.

In a continuously circulating atmosphere where no condensation takes place, the ratios of molar densities of all components at all heights are equal to their mean atmospheric values. This is due to the fact that the diffusional velocities that could restore Boltzmann distributions as dictated by molar masses of particular air components are small compared with the dynamic velocities of the air flow. According to observations, mixing ratios of the non-condensable air components and the molar mass of dry air are the same at all heights in the troposphere (McEwan and Phillips, 1975).

Consequently, the process of condensation in the rising air should be reflected in the changes of the ratio $\gamma \equiv N_{v} / N$ (relative molar density of water vapour). Saturated pressure of water vapour diminishes with decreasing temperature. Therefore, if the air moves along a temperature gradient towards the lower temperature, such a movement can be associated with condensation. However, there can also be density changes unrelated to condensation: to estimate the condensation rate, such reference density changes should be subtracted from the overall density change of saturated vapour. Molar rate $S$ of condensation per unit volume $\left(\right.$ mole $\left.\mathrm{m}^{-3} \mathrm{~s}^{-1}\right)$ can be written as

$$
\begin{aligned}
& S=\boldsymbol{w}_{0} \nabla N_{v}-\gamma \boldsymbol{w} \nabla N+\gamma(N / T) \boldsymbol{u} \nabla T, \\
& \gamma \equiv \frac{N_{v}}{N}=\frac{p_{v}}{p}, \quad \nabla \equiv \frac{\partial}{\partial x} \boldsymbol{i}+\frac{\partial}{\partial y} \boldsymbol{j}+\frac{\partial}{\partial z} \boldsymbol{k} .
\end{aligned}
$$

Here, $\boldsymbol{i}, \boldsymbol{j}$ and $\boldsymbol{k}$ are the unit orthogonal vectors specifying the directions of coordinate axes $x, y$ and $z$, respectively, $\boldsymbol{w}_{0}$ is the velocity vector directed along the temperature 
gradient towards the lower temperature, $\boldsymbol{w}$ is the vertical velocity and $\boldsymbol{u}$ is the horizontal velocity.

In the general case, velocities $\boldsymbol{w}_{0}$ and $\boldsymbol{w}$ are not parallel and the isothermal plane is not horizontal. Let $\boldsymbol{u}_{0}$ be the velocity vector in the isothermal plane perpendicular to the temperature gradient and to velocity $\boldsymbol{w}_{0}$. We choose axes $x_{0}, x, z_{0}$ and $z$ to be directed along velocities $\boldsymbol{u}_{0}, \boldsymbol{u}, \boldsymbol{w}_{0}$ and $\boldsymbol{w}$, respectively (then, $x_{0} y_{0}$ is the isothermal plane and $x y$ is the horizontal plane). The magnitudes of these velocities are related as follows:

$$
\begin{aligned}
& w_{0}=w \cos \alpha-u \sin \alpha=w-u \alpha+o\left(\alpha^{2}\right), \\
& u_{0}=u \cos \alpha+w \sin \alpha=u-w \alpha+o\left(\alpha^{2}\right) .
\end{aligned}
$$

Here, $\alpha$ is the angle between the isothermal and the horizontal plane; $\alpha=0$ for a horizontal isothermal surface, then $u_{0}=u$ and $w_{0}=w$. Tangent of this angle is equal to the ratio of horizontal to vertical temperature gradients and is normally very small.

The physical content of equation (2) consists in the fact that condensation is associated with air movement along the temperature gradient (no condensation occurs in the isothermal plane); that there is a hydrostatic equilibrium distribution of air density in the vertical dimension, and that in the absence of condensation the horizontal pressure gradient would be zero. The term $\gamma \boldsymbol{w} \nabla N$ subtracted from the total density change $\boldsymbol{w}_{0} \nabla N_{v}$ describes the equilibrium gravitational expansion of water vapour that is unrelated to condensation. Likewise, term $(-\gamma N / T u \nabla T)$ subtracted from the total density change describes the equilibrium density change of water vapour that would have occurred in the horizontal plane in the absence of condensation. Assuming that in the absence of condensation $\partial p / \partial x=0$, we have $\partial N / \partial x=\partial(p / R T) / \partial x=-(\partial T / \partial x) N / T$. In other words, the horizontal temperature gradient $\partial T / \partial x$ contains the information about the equilibrium density distribution and can be used as the reference term in equation (2). Such horizontal equilibrium is manifested in the fact that air pressure at the surface is globally unrelated to surface temperature, i.e., it is close to standard atmospheric pressure both in high and in low latitudes.

Since condensation rate $S(2)$ is a function of molar (not mass) densities, it is convenient and physically transparent to write the continuity equation for ideal gas in terms of molar densities as well. The stationary continuity equation is

$$
\nabla N \boldsymbol{v}=N \nabla \boldsymbol{v}+\boldsymbol{v} \nabla N=S, \boldsymbol{v} \equiv \boldsymbol{u}_{0}+\boldsymbol{w}_{0}=\boldsymbol{u}+\boldsymbol{w} .
$$

Taking into account that $N=N_{d}+N_{v}$ and the conservation of mass of dry air,

$$
\nabla N_{d} \boldsymbol{v}=0=N_{d} \nabla \boldsymbol{v}+\boldsymbol{v} \nabla N_{d},
$$

we obtain from equation (6):

$$
\nabla N_{v} v=N_{v} \nabla \boldsymbol{v}+v \nabla N_{v}=S .
$$

We consider water vapour to be saturated, such that its partial pressure and molar density do not change in the isothermal plane: $\boldsymbol{u}_{0} \nabla N_{v}=\boldsymbol{u}_{0} \nabla T=0$ and $\boldsymbol{v} \nabla N_{v}=\boldsymbol{w}_{0} \nabla T$. Using expression for $S(2)$ and multiplying both parts of equation (8) by $\gamma^{-1}$, we have:

$$
\boldsymbol{w} \nabla N-N \boldsymbol{u} \nabla T / T+N \nabla \boldsymbol{v}=0 .
$$

Using this result, we have from equation (6):

$$
\boldsymbol{u}(\nabla N+N(1-\gamma) \nabla T / T)=\boldsymbol{w}_{0} \nabla N_{v}-\gamma \boldsymbol{w} \nabla N .
$$


Going over from $N$ and $N_{v}$ to $p$ and $p_{v}$ using equations (1) and (6), we finally obtain:

$$
\boldsymbol{u} \frac{\nabla p}{p}=\gamma\left(\boldsymbol{w}_{0} \frac{\nabla p_{v}}{p_{v}}-\boldsymbol{w} \frac{\nabla p}{p}\right) .
$$

Taking into account that $w_{0} \approx w-\alpha u$ (4) and recalling that $\boldsymbol{u}_{0} \nabla p_{v}=0$, we can write equation (11) as

$$
\begin{aligned}
& -\frac{\partial p}{\partial x}=\frac{w}{u} \frac{p_{v}}{h_{\gamma 0}} \approx \frac{w}{u} \frac{p_{v}}{h_{\gamma}}\left(1-\frac{h_{\gamma}}{h_{v}} \frac{u}{w} \alpha\right), \\
& h_{\gamma 0}^{-1} \equiv \frac{w_{0}}{w} h_{v}^{-1}-h^{-1}, \quad h_{\gamma}^{-1} \equiv h_{v}^{-1}-h^{-1}, \quad h_{v}^{-1} \equiv-\frac{1}{p_{v}} \frac{\partial p_{v}}{\partial z_{0}}, \quad h^{-1} \equiv-\frac{1}{p} \frac{\partial p}{\partial z} .
\end{aligned}
$$

Here, scale height $h$ is determined by the condition of hydrostatic equilibrium (see Appendix); scale $h_{v}<h$ is determined by the Clausius-Clapeyron equation that governs the dependence of saturated vapour pressure on temperature (see Appendix). The approximation in equation (12) is valid to the accuracy of the ratio $\left(h_{\gamma} / h_{v}\right)(u / w) \alpha<<1$. We discuss the physical meaning of the obtained result in the following section.

\section{Acceptor and donor regions of condensation-induced circulation}

As the mass of dry air components is conserved, so dry air flows over closed trajectories. It is convenient to term the low-pressure region that the air streams towards as an acceptor region. Low-level air is 'sucked' into an acceptor region from neighbouring areas with higher surface pressure - such an area can be conveniently termed a donor region.

Let us now consider a large-scale stationary circulation where the air flux enters the acceptor region moving along the horizontal $x$-axis via a cross-section of area approximately equal to $D h_{\gamma}$ and leaves the circulation area as it moves vertically along the $z$-axis via a cross-section of area $D L$. Here, $L$ is the length of the acceptor region and $D$ is the width of the acceptor region counted along the $y$-axis perpendicular to the air flow. Taking into account that the number $n_{\text {in }}$ of air moles that enter the acceptor area differ from the number $n_{\text {out }}$ of air moles leaving the acceptor region by a relatively small number of moles of condensed water vapour, $n_{\text {in }}-n_{\text {out }} \sim m_{\text {in }}<<n_{\text {in }}$, to the accuracy of $\gamma<<1$ we can write $D h, \mu=D L w$ or

$$
\frac{w}{u}=\frac{h_{\gamma}}{L}
$$

This equation represents the continuity equation for air in the integral form that is valid at small $\gamma$. At constant velocities $u$ and $w$, this relationship is conserved everywhere in the acceptor region, see equation (A3) in Appendix. Putting (14) into the approximate relationship in equation (12), we have:

$$
-\frac{\partial p}{\partial x} \approx \frac{\Delta p}{L}\left(1-\frac{L}{h_{v}} \alpha\right), \quad \Delta p \equiv p_{\gamma}=p_{v} .
$$


This result shows that maximum horizontal pressure drop that can be observed between the donor and acceptor regions on an isothermal surface (when $\partial T / \partial x=0$ and $\alpha=0$ ) is equal to the partial pressure of water vapour $p_{v}$. This corresponds to the situation when all the vapour transported from the donor region has been used up (condensed) as the air travels along the temperature gradient in the acceptor region.

Equation (15) contains an essential assumption, namely that the height of the donor and acceptor regions is equal to the scale height $h_{\gamma}$ of the condensation process. This assumption should be valid for cases when $h_{\gamma}$ approximately coincides with the atmospheric scale height $h$. This is the case for high temperatures characteristic of tropical regions (see Figure 1 in Makarieva et al. (2010)). In this case, equation (15) illustrates that a positive horizontal temperature gradient, $\partial T / \partial x>0$ and $\alpha \approx(\partial T / \partial x) /(\partial T / \partial z)<0$, diminishes the horizontal pressure drop, while a negative horizontal temperature gradient increases it. The magnitude of the temperature correction decreases with diminishing length $L$ of the circulation pattern. With temperature increasing by $\Delta_{x} T=5 \mathrm{~K}$ over length $L \sim 10^{3} \mathrm{~km}, \partial T / \partial x=\Delta_{x} T / L$, and decreasing by $\Delta_{z} T=50 \mathrm{~K}$ over height $h_{\gamma} \sim 10 \mathrm{~km}, \partial T / \partial z=\Delta_{z} T / h_{\gamma}$, we have $\left(L / h_{\gamma} \alpha\right)=\Delta T_{x} / \Delta T_{z}=0.1<<1$ at $h_{\gamma} \sim h_{v}$. This estimate illustrates the relatively minor role of typical horizontal temperature gradients in modifying condensation-induced pressure differences.

A detailed analysis of temperature effects is beyond the scope of this paper. Here, we only note two essential points. First, the release of latent heat during condensation can modify an external temperature gradient, as discussed by Makarieva et al. (2010) in the context of condensation-induced dynamics. The drier air that descends adiabatically warms more rapidly with decreasing altitude than the moist air cools with increasing altitude as it ascends. This causes the area of descent to be warmer in the lower atmosphere than the area of ascent (acceptor region) due to transport of latent heat released upon condensation to the donor region.

In circulation patterns with intense condensation, e.g., in hurricanes, this effect can amount to a horizontal temperature difference at the Earth's surface of $2-3^{\circ} \mathrm{C}$ (see Figure 4(c) of Montgomery et al., 2006), with the ascending air being significantly colder than the air that descends in the eye and outside the hurricane. Additionally, higher evaporation in the acceptor region compared with the donor region can make the land surface cooler in the acceptor region by harbouring a greater part of the incoming solar flux as latent heat. Second, at a given evaporation rate, vertical velocity $w$ in a stationary circulation pattern is inversely proportional to the partial pressure of water vapour (Makarieva and Gorshkov, 2007), as the total flux of latent heat associated with the adiabatic ascent is limited by solar power. This should be taken into account when analysing high latitude regions with significantly different height scales $h$ and $h_{\gamma}$

Here, we confine our consideration to the case when the horizontal plane is isothermal, $\partial T / \partial x=0$. This case is both visually transparent and physically important, as it highlights the difference between the conventional paradigm of pressure gradients associated with differential heating of the Earth's surface (e.g., Gill, 1982, p.24) and the condensation-induced pressure gradients that can be realised on an isothermal surface.

Equations (14) and (15) are valid for a bunch of streamlines that enter the circulation area in the horizontal direction and leave it in the vertical direction. The number of horizontal streamlines that come from the donor region and reach distance $x$ decreases as the air travels inside the acceptor region. This decrease occurs at the expense of those streamlines that have left the acceptor region in the vertical direction. Velocities $u$ and $w$ 
and pressure gradient are the same for each streamline; the mean horizontal velocity in the total column linearly decreases, see Figure 2 and Appendix. In Figure 2, in reality, the mean height where the air starts ascending does not depend on $x$ (this would correspond to a random rearrangement of the streamlines in the figure with respect to where they begin to ascend). The height where the air ascends conserving positive horizontal velocity is of the order of, but actually less than, $h_{\gamma}$. The value of $h_{\gamma}$ characterises the total height of the circulation pattern, including the part of the acceptor region where the air still ascends but the direction of horizontal flow has reversed (not shown in the figure).

Figure 2 A scheme of air streamlines above the forest-covered acceptor region of length $L$ and height $h_{\gamma}$ The continuity equation is $u h_{\gamma}=w L(14)$. The regular horizontal spacing of the vertical parts of the streamlines corresponds to constant precipitation rate everywhere in the forest-covered region. Total flux of water vapour that reaches point $x$ is proportional to the number of horizontal lines crossing the vertical cross-section of the air flow at point $x$. This flux linearly decreases with growing $x$ as the air flow penetrates further inland (the dashed line). Closed curves with arrows show turbulent eddies (horizontal roll vortices) that are responsible for local evaporation and condensation not related to large-scale moisture transport. Rotation axis of the eddies can be parallel to the direction of the main flow

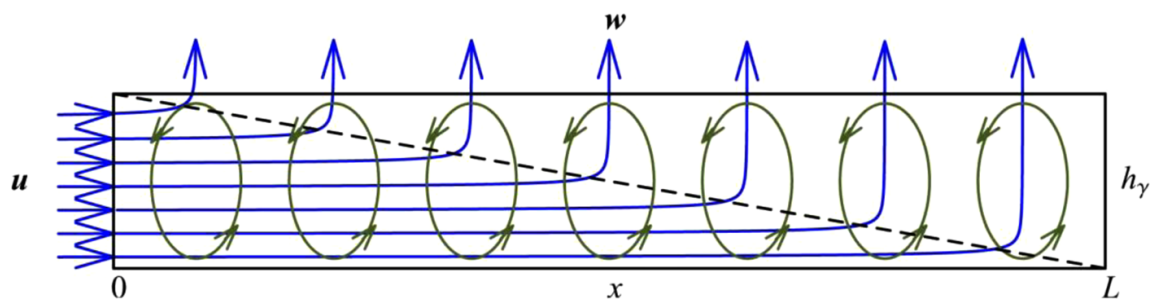

From equation (14), it is clear that $p_{v}=\Delta p$ represents a store of potential energy that is converted to the kinetic energy of moving air masses as the water vapour condenses. By integrating equation (14) at $\alpha=0$, we obtain the following expression for potential energy $\Pi(x)=p(x)$ that is defined to the accuracy of a constant term:

$$
\Pi(x)=p(x)=p(0)-\frac{\Delta p}{L} x .
$$

In the incoming air flow at $x=0$, water vapour is present everywhere in that part of the atmospheric column that is affected by the circulation. This water vapour is the water vapour imported from the donor region. At $x=L$, the imported water vapour has been completely used up. The remaining water vapour is the water vapour evaporated locally in the acceptor region (see Appendix, equation (A5)).

In a pure vapour atmosphere with a sufficiently large vertical lapse rate of air temperature, a unidirectional upward air flow would be possible, with saturated vapour ascending along a vertical gradient of decreasing partial pressure governed by the decreasing temperature. In this case, it would rain continuously everywhere over the ocean. Such a unidirectional gas flow is realised in heat pipes - technological devices that employ the partial pressure gradient of the saturated vapour to effectively transfer heat (Grover et al., 1964). The rapid transport of heat along the pipe is due to the difference in partial pressures of saturated vapour within the pipe, as one end of the pipe is warm, another is cold; this pressure gradient causes the vapour to flow very rapidly along the tube from the hot end to the cold. 
But in the presence of non-condensable components, a unidirectional flow is not possible. The oceanic surface of Earth is dynamically mapped into acceptor regions with moist adiabatic ascent, condensation, cloud formation and precipitation and donor regions where the air adiabatically descends and condensation and precipitation are absent. Unlike the pure vapour atmosphere considered earlier, it does not rain everywhere, but only in acceptor regions. The fact that the mean global cloudiness is reasonably close to 50\% (Mokhin and Schlesinger, 1994) indicates that acceptor and donor regions are on global average of approximately the same size. The donor and acceptor regions are connected by horizontal air flows: one in the lower atmosphere brings moist air from the donor to acceptor region, the other in the upper atmosphere returns dry air from the acceptor to the donor region.

Evaporation is present in both donor and acceptor regions, while condensation and precipitation occur in the acceptor region only. Water vapour that evaporated in the donor region is imported into the acceptor region where it condenses and precipitates together with the locally evaporated moisture. Consequently, precipitation in the acceptor region is always higher than local evaporation. Precipitation exceeds evaporation by the amount of water vapour imported from the donor region. If the donor and acceptor regions feature approximately similar size and have approximately equal evaporation rates, precipitation in the donor region will be about twice the value of local evaporation. When the acceptor region is located on land, the difference between precipitation and local evaporation will return to the ocean as runoff.

The co-existence of hydrosphere, water vapour and non-condensable gases in the gravitational field necessitates condensation-induced circulation and the division of the atmosphere into the regions with and without precipitation. Unlike the situation in heat pipes, where the temperature gradient responsible for condensation has to be maintained externally, so imposing limits on pipe performance, in condensation-induced circulations cooling of moist air occurs simply due to its vertical movement, i.e., there is a positive feedback between condensation and velocity. The nature of this feedback is such that the condensation-induced circulation cannot be stopped. However, in principle, through reafforestation, it should be possible to make the division of acceptor and donor areas stationary and enlarge their linear size up to several thousand kilometres, as we discuss in Section 6 .

\section{Evaporation maintains horizontal pressure gradients induced by condensation}

The continuity equations (11) and (12) can be written in a compact form using the definition of $\gamma(3)$ :

$$
u \frac{\partial \ln \gamma}{\partial x}=-w \frac{\partial \gamma}{\partial z}, \quad u \frac{\partial \ln \gamma}{\partial x}=-\frac{1}{p} \frac{\partial p}{\partial x}, \quad \frac{\partial p_{v}}{\partial x}=0 .
$$

It follows from this equation that the adiabatic ascent of moist air accompanied with condensation, $-\partial \gamma / \partial z>0$, can only occur if there is an input of evaporated water vapour into the horizontal air flow, i.e., $\partial \gamma / \partial x>0$ (Makarieva et al., 2010).

Evaporation is a surface-specific process anchored predominantly to the Earth's surface, while condensation is a volume-specific process that occurs in the 
atmospheric column. Therefore, while evaporation adds water vapour to the atmosphere, the evaporation flux is not added to the $S$ term in equation (2). The latter equation describes only those phase transitions (condensation) that occur within the atmospheric column. The evaporated moisture is added to any given air volume by means of turbulent mixing, with turbulent eddies exchanging air parcels between the saturated layer near the land or ocean surface and the rest of the column, Figure 2. Such eddies change the mixing ratio of water vapour along the horizontal axis.

Equation (17) illustrates the necessity of continuous evaporation from the Earth's surface for the maintenance of a horizontal pressure gradient. As the air moves horizontally along the gradient of decreasing pressure, the partial pressure of water vapour decreases together with total air pressure. Suppose the land surface is dry and there is no vapour input into the horizontally moving air. Water vapour becomes unsaturated as it moves. As far as the adiabatic ascent of unsaturated air is not accompanied by condensation, the condensation-induced pressure gradient is no longer sustained. The horizontal air flow ceases and the circulation stops. Evaporation from the Earth's surface keeps the water vapour saturated in the horizontal air flow such that condensation in the adiabatically ascending air is sustained.

Evaporation is maintained by solar energy and cannot exceed a certain maximum rate when all solar power is used up on evaporation. Such maximum evaporation power, when globally averaged, constitutes about $2 \mathrm{myear}^{-1}$, while the observed global evaporation rate is about $1 \mathrm{~m} \mathrm{year}^{-1}$ (L'vovitch, 1979). This illustrates the corridor available for an increase in evaporation rate by forests compared with open water surfaces. The condensation rate depends on vertical velocity and is not related to evaporation rate. It can be zero in the donor region and significantly exceed local evaporation rate in the acceptor region, as is the case in hurricanes and tornadoes. With increasing evaporation, the condensation rate grows more rapidly than evaporation does. The acceptor region is, therefore, always the region where evaporation is higher, i.e., natural forest (acceptor) compared with the ocean (donor).

Air circulation in the acceptor region is a superposition of the large-scale incoming flow and local turbulent eddies, Figure 2. These eddies contribute to the local evaporation-condensation cycle; they have horizontal size of the order of atmospheric height $h$ and wind velocities $u \sim w$ (see Appendix). If there is no large-scale flow, the local eddies associated with local evaporation and condensation do not move as a whole along the land surface. They are oriented chaotically and, due to such spatial randomness and the absence of a preferential direction of movement, can occasionally grow in size producing squalls with $u>>w$ and merge into cyclones, hurricanes or tornadoes, accompanied by floods and subsequent droughts. When a large-scale flow from the donor to acceptor region is present, eddies start moving together with the flow. This makes their spatial distribution and direction of rotation more ordered (e.g., via formation of the so-called 'cloud streets'), Figure 2, and prevents eddies from merging into larger and more violent circulation patterns thus stabilising the circulation.

\section{Stabilising the continental moisture supply: the role of friction}

One might describe forests as caught up in a 'tug of war' with the ocean to draw in moist air. Second, it is necessary to stabilise the incoming moisture flux. Indeed, the store of potential energy accumulated in the condensational pressure gradient could be released in 
an avalanche-like manner, producing violent winds, uncontrollably strong precipitation and floods. During floods, the bulk of precipitated water is lost as runoff, instead of being used for transpiration, and so soil erosion arises. Unlike condensation, which can occur in a burst-like manner, evaporation is normally a gradual process limited by the absorbed flux of solar radiation. Thus, after a flood, as the atmosphere slowly replenishes its water vapour, a drought may follow.

To achieve a safe balance between the Scylla and Charybdis of floods and droughts, it is important to mitigate the development of catastrophically strong winds. This form of climate chaos can be avoided if some part of the potential energy available upon condensation is lost to friction rather than totally converted into the kinetic energy of wind. Here, we consider friction effects in the biotic pump mechanism.

In the absence of friction, the release of potential energy associated with the condensation-induced pressure gradient (12) would accelerate air up to maximum velocity $u_{c}$ defined by the equation $\Delta p=\gamma p=\rho u_{c}^{2} / 2$. All potential energy is fully converted to kinetic energy. At $\gamma=0.04, T=30^{\circ} \mathrm{C}, p=10^{5} \mathrm{~Pa}$, we have $u_{c}=80 \mathrm{~m} \mathrm{~s}^{-1}$, which is a hurricane wind speed (Makarieva and Gorshkov, 2009b). (In tornadoes due to this radial convergence, wind velocities may theoretically exceed $u_{c}$ and reach $130 \mathrm{~m} \mathrm{~s}^{-1}$.)

The major friction force per unit area of the Earth's surface, which prevents air from full acceleration, is the friction force $F_{f}$ that can be called gravitational. It is proportional to the weight of atmospheric column $F_{f}=\mu \rho g h=\mu p_{s}, \mu=z_{T} / h \sim 10^{-5}$ (Makarieva and Gorshkov, 2009a). Here, $p_{s}$ is the surface pressure, $z_{T} \sim 0.1 \mathrm{~m}$ is the scale height of surface roughness (it is proportional to the height of vegetation cover, oceanic waves, etc.) and $h \sim 10 \mathrm{~km}$ is the scale height of the atmosphere. The same form of gravitational friction that is independent of air velocity, $\mu g h$, is due to frictional dissipation of liquid drops precipitating or suspended in the atmosphere. In this case, $\mu$ is equal to the relative volume occupied by the drops, $\mu \sim \gamma\left(w / w_{b}\right) \sim 10^{-5}$, where $w_{b}$ is the mean downward velocity of the drops, $w / w_{b} \sim 10^{-3}$. The gravitational friction force does not depend on air velocities $u$ or $w$ and can be represented as $\mu \rho g h=\rho u_{g^{*}}^{2}$, where $\mu=u_{g^{*}}^{2} / g h$ is the Froude number, $u_{g^{*}} \sim 1 \mathrm{~m} \mathrm{~s}^{-1}$ has the meaning of the rotation velocity of turbulent eddies that separate from the main air flow due to the gravitational friction. The gravitational friction force $\rho u_{g^{*}}^{2}$ is more than an order of magnitude larger than the force of aerodynamic friction $c_{D} \rho u^{2}=\rho u_{*}^{2}, c_{D} \approx h_{\gamma} / L=w / u \sim 10^{-3}\left(u_{g^{*}}^{2} \sim 30 u_{*}^{2}\right)$. The latter force is usually taken into account in the Navier-Stokes equations and the formulation of Reynolds stress but is insufficient to compensate for the condensation-induced pressure gradient (Makarieva and Gorshkov, 2009a).

Friction force per unit air volume, $f_{f}$, averaged over the atmospheric column where the circulation takes place is equal to the surface-specific friction force $F_{f}=\mu \rho g h=\mu p_{s}$, where $p_{s}$ is air pressure at the land surface, divided by the scale height $h_{\gamma}$ of the condensation-induced circulation: $f_{f}=F_{f} / h_{\gamma}$ The evaporative-condensational force acting per unit air volume is $f_{E}=F_{E} / h_{\gamma}$ where $F_{E}=\Delta p=\gamma_{s} p_{s}$ is the evaporative-condensational force per unit area of the Earth's surface, which coincides with the total horizontal pressure drop in the circulation area. The air flow does not accelerate if the amounts of work performed by the two forces per unit time, i.e., their powers, coincide:

$$
f_{E} w=f_{f} u \text {. }
$$


Taking relationship (14) into account, this equality can be written as

$$
\frac{L}{h_{\gamma}}=\frac{u}{w}=\frac{\gamma}{\mu} \equiv K \sim 10^{3} .
$$

The obtained value of $K$ corresponds to the mean value of the meridional wind velocity $u \approx 2 \mathrm{~m} \mathrm{~s}^{-1}$ and vertical velocity $w \approx 2 \mathrm{~mm} \mathrm{~s}^{-1}$ (e.g., Rex, 1958). At the known value of $\gamma$, equation (19) yields physically sound $\mu$ values that agree satisfactorily with independent theoretical estimates (Makarieva and Gorshkov, 2009a). Note that the estimate (19) is valid to the accuracy of the order of magnitude.

Equation (19) illustrates that to ensure sufficient friction and prevent the development of high wind speeds and large fluctuations of the continental moisture supply, the forest cover must feature a very extensive length comparable with the linear size of the continents, $L \sim 10^{3} h_{\gamma}$. In this case, the regular air drawn by the biotic pump prevents chaotic condensation. The biotic pump does not allow hurricane-force winds to develop either in the acceptor region where it works, or in the adjacent donor region. For example, no hurricanes occur in the Atlantic ocean where it borders with natural forests of the Amazon and Congo river basins.

In Section 3, we derived the expression for the horizontal pressure gradient induced by condensation based on the continuity equations for dry and moist air. This gradient (12) is a function of the gradient of the saturated pressure of water vapour dictated by temperature and of air velocities $u$ and $w$. These velocities, in their turn, are determined by air pressure gradients and friction losses including friction due to precipitation (formation and falling of droplets). The vertical gradient of water vapour does not depend on whether precipitation has taken place or not: vapour pressure diminishes immediately upon condensation. In contrast, air velocities are affected by the process of precipitation, which represents a significant source of friction. The smaller the drops, the longer they remain in the atmosphere, the greater friction losses are caused and the smaller velocities result. Equation (12) for the horizontal pressure difference takes into account the properties of both precipitation and condensation.

A certain part of condensed moisture representing the smaller drops with the lower terminal velocity is carried upwards by the ascending air flow; such drops can leave the acceptor area and precipitate elsewhere. However, the amount of non-precipitated liquid that is routinely sustained in the atmosphere represents a tiny fraction of total precipitable water content. The amount of liquid water stored in the atmosphere per unit surface area is around $50 \mathrm{~g} \mathrm{~m}^{-2}$ (Wood et al., 2002), while a typical precipitable water content can be around $150 \mathrm{~kg} \mathrm{~m}^{-2}$ (Duan et al., 1996).

So how is the circulation induced by condensation affected if moisture condensed in the acceptor region does not precipitate but is transported by the ascending air flow back to the donor region? This can be explained as follows: once the characteristics of droplet formation are accounted for as friction losses, the spatial localisation of precipitation over the donor or acceptor region will not affect the circulation pattern. That is, the net flux of water from the donor to acceptor region will be affected by where the moisture precipitates, but wind speeds and pressure gradients will not. We emphasise this point: the proposed condensation-induced atmospheric dynamics is not just a mechanism that transports moisture from ocean to land and runs the water cycle on land; it is a general physical mechanism that generates winds, which, under certain circumstances, can also transport moisture to land. 
More importantly, transport of a major part of condensed moisture back to the donor region never actually happens. If this was the case, the amount of liquid and vapour in the atmosphere would be approximately equal: moisture would travel as vapour to the acceptor region in the lower atmosphere and return as liquid to the donor region in the upper atmosphere; the runoff would be absent. Thus, $\mathrm{H}_{2} \mathrm{O}$ molecules would spend approximately equal time in the atmosphere in the form of vapour and liquid. In reality, the amount of liquid moisture in the atmosphere and, hence, the time moisture spends in the atmosphere in the form of liquid are three orders of magnitude smaller than the amount of vapour and the time moisture spends in the form of vapour, respectively. Hence, most moisture precipitates in the acceptor region where the moist air ascends and vapour condenses, with spatial fluctuations of this process dictated by local turbulent eddies, Figure 2. Transport of liquid moisture from the acceptor to donor region predominantly occurs as runoff.

\section{The biotic pump of natural forests vs. non-forest vegetation}

The forest moisture pump is peculiar in that a strict biological control is exerted over the processes of evaporation (both transpiration and intercept) and condensation. Given a very large evaporative surface of leaves compared with an open water surface, and the capability of plant control of transpiration by regulating stomata openings, a forest is able to sustain evaporation and condensation at an optimal level determined by the biological demand for soil moisture. The large value of tree height keeps the horizontal velocity $u$ of air flow relatively constant, minimising the possibility of hurricane and tornado formation. The year-round sustainability of condensation and therefore precipitation and soil moistening is associated with the observed constancy of river runoff in the undisturbed forest-covered river basins (Makarieva and Gorshkov, 2007; Makarieva et al., 2009), Figure 3. By pumping enormous amounts of atmospheric moisture from the ocean, a forest regulates precipitation to be spatially uniform over the entire catchment; moisture is then returned to the ocean in the liquid state as runoff. Forest climate control by precipitation prevents moisture shortage and droughts, as well as excessive precipitation and floods. The latter is particularly important near the coast where the horizontal ocean-to-land moisture flux is maximum.

Let $F(x)$ be the horizontal moisture flux equal to the amount of atmospheric moisture passing inland across a unit horizontal length perpendicular to the stream line per unit time at distance $x$ from the ocean, dimension $\mathrm{kg} \mathrm{H}_{2} \mathrm{O} \mathrm{m}^{-1} \mathrm{~s}^{-1}$. For a river basin covered by natural forest, we have

$$
\frac{\mathrm{d} F(x)}{\mathrm{d} x}=R=\mathrm{const}, \quad F(x)=F(0)-R x, \quad F(0)=R L, \quad 0 \leq x \leq L,
$$

where $R$ is the river runoff per unit surface area, $L$ is the linear size of river basin, which, as estimated in the previous section, can reach several thousand kilometres, Figure 3.

Scarce and low vegetation with relatively small leaf surface area can develop evaporation fluxes exceeding those of the open ocean only under some special conditions, in particular, in summer when the available solar radiation is largest. During such periods as, for example, during the so-called wet monsoon, flux $F$ of moist air propagates from the ocean to land. However, in this case, the river runoff, which is 
proportional to precipitation not regulated by scarce vegetation, changes proportionally to flux $F$. This leads to an exponential decline of runoff $R(x)$ and precipitation with distance $x$ from the ocean, with the excessive amount of precipitation causes floods in the vicinity of the coastline, Figure 3:

$$
R(x)=\frac{\mathrm{d} F(x)}{\mathrm{d} x}=-\frac{1}{\lambda} F(x), \quad \frac{\mathrm{d} F(x)}{\mathrm{d} x}=\left(\frac{\mathrm{d} F(x)}{\mathrm{d} x}\right)_{0} \exp \left(-\frac{x}{\lambda}\right) .
$$

Here, $\lambda$ is the exponential scale length of precipitation decline in non-forested regions. It is of the order of $600 \mathrm{~km}$ on a global average (Makarieva and Gorshkov, 2007; Makarieva et al., 2009). The exponential decline of runoff and precipitation testifies to the inability of scarce and low vegetation to control the runoff and precipitation, Figure 3. This turns the hydrological cycle into a sequence of droughts and floods accompanied by extreme winds. Notably, biological systems such as savannas are themselves unstable. They either recover to the natural forest in the absence of anthropogenic disturbance or ultimately degrade to deserts if the anthropogenic disturbance persists. The demise of the Peruvian Nazca civilisation as a result of large-scale forest logging to make space for agricultural lands (Beresford-Jones et al., 2009) is readily explained by the biotic pump theory. The theory provides a general physical platform for analysing the apparently critical role of land use and cover changes in global change (e.g., McAlpine et al., 2009) and regional land cover impact on air circulation (Chikoore and Jury, 2010).

The Siberian forests, including the forests of the Far East, are unique in that they draw moisture from three oceans: the Atlantic, Arctic and Pacific. This has allowed these forests to persist even after European forests were largely destroyed by humans. By contrast, the extant continental forests of now desertified regions in Australia, Arabia and the Sahara did not survive the elimination of the coastal forest zone. Siberan forests, with unimpeded access to the moisture stores of the Arctic and Pacific oceans, were able to continue drawing moisture from the Atlantic Ocean past the largely deforested Europe. The zone of low pressure maintained over forested Siberia has contributed to the persistence and regularity of moist western winds over Europe. Europe has not become a desert due to the biotic pump function of Siberian and remaining Eastern European forests.

However, the recent elimination of natural forests over large areas of Russia, including Siberia, has made the western moist air flow irregular. The unprecedented heat wave in southwestern Russia in the summer of 2010 (http://earthobservatory.nasa.gov/ IOTD/view.php?id=45069) is an example of weather extremes caused by breakdown of the biotic pump. The climatically affected area, with monthly temperatures somewhere exceeding long-term averages by several degrees, turned into a typical donor region: all the locally evaporated moisture was taken away by low-level air flow to neighbouring regions causing floods in other European and Asian countries. The air was descending in the deforested dry continental interior and ascending in regions adjacent to the ocean, with the donor continental region being about $10^{\circ}$ warmer at land surface than the acceptor region. This observation illustrates the greater importance of condensation-induced dynamics for driving air motions when compared with the more conventional theory of differential land surface heating by solar radiation. 
Figure 3 Biotic pump of atmospheric moisture in river basins covered by natural forests (black symbols) when compared with disturbed and unforested regions (open symbols) (after Makarieva et al. (2009)). Shown is the mean annual precipitation on land associated with large-scale regional atmospheric circulation patterns as dependent on distance from the ocean. Numbered arrows on the map indicate which regions were considered. (a), (b), (c): data for the world's largest river basin covered by natural forests. In the basins of world's largest rivers - Amazon and Congo - precipitation is twice higher than over the ocean and does not decrease with growing distance from the ocean. Precipitation in the basins of the northern rivers that flow from the south grow with distance from the ocean proportionally to the increase in solar radiation.

(d), (e), (f): precipitation over non-forested areas decline exponentially with distance from the ocean
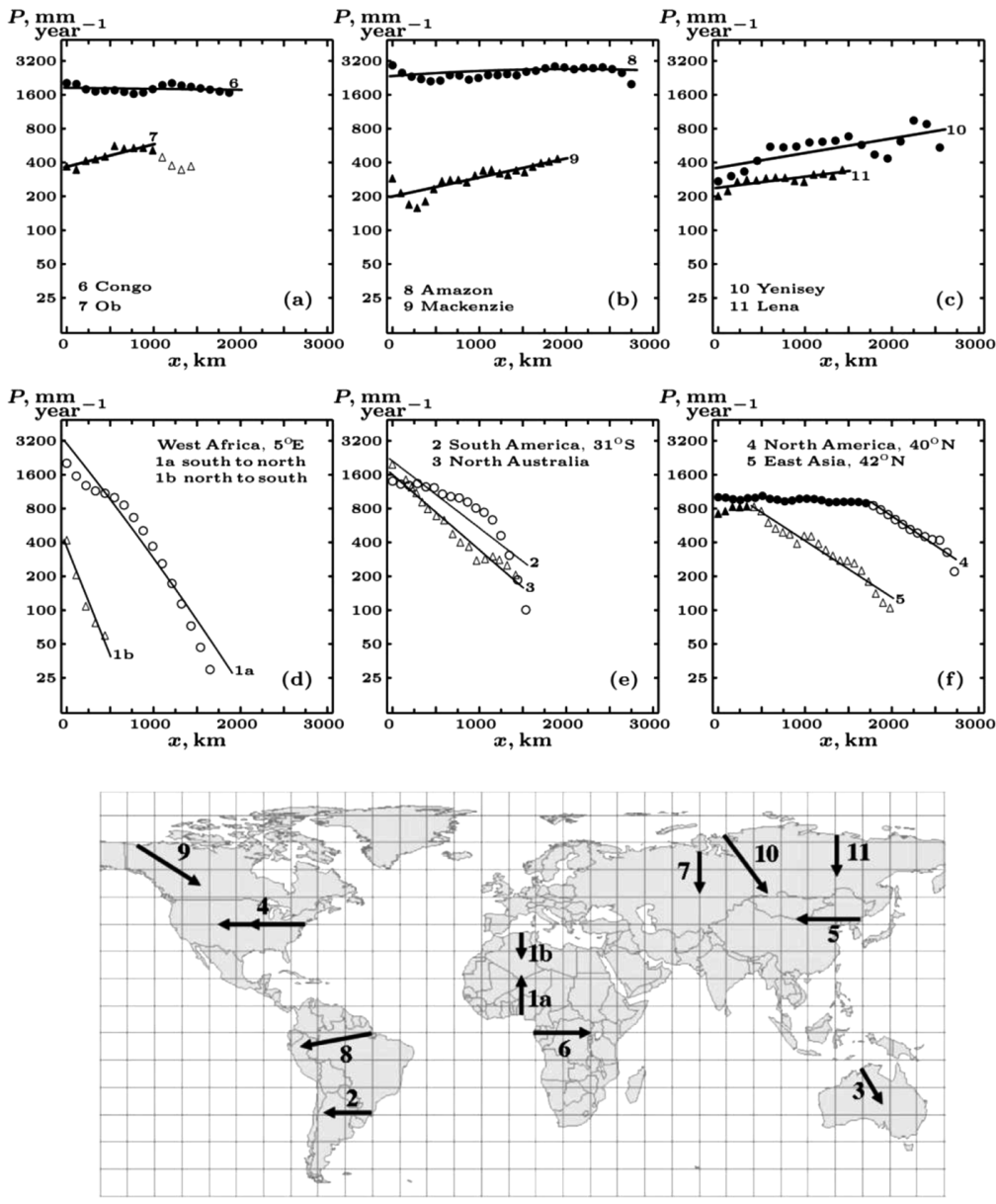

\section{Summary and conclusions}

In this paper, we have summarised the current status of the biotic pump theory, which quantitatively describes the physical principles allowing the continents to get moistened and inhabited by life. It has been noted previously that the non-equilibrium drop of water vapour partial pressure is translated into horizontal gradients of air pressure 
(Makarieva and Gorshkov, 2007, p.1023). The condensation-induced pressure gradient was later estimated for compact horizontally isothermal mobile circulation patterns like hurricanes and tornadoes on the basis that the rate of pressure change along the horizontal axis should be equal to the rate of pressure change along the vertical axis (Makarieva and Gorshkov, 2009b; Makarieva et al., 2010). Here, we presented a detailed derivation of the condensation-induced horizontal pressure gradient for the general case of a non-isothermal surface. We then considered the physically important isothermal case in greater detail and illustrated the role of evaporation on land for maintaining the condensation-induced air circulation. We discussed the role of friction, including friction related to precipitation, in stabilising the condensation-induced air flow. We finally reviewed data on spatial precipitation patterns as influenced by forest vs. non-forest land cover (Makarieva et al., 2009).

While scientists have recognised the principles of condensation-induced dynamics only recently, life apparently 'discovered' them about half a billion years ago. These principles underlay the formation of a continuous forest cover that colonised practically all land. Prior to the evolutionary appearance of trees and forest ecosystems, land had been a lifeless desert. The mean lifespan of biological species, which in the stationary state is equal to the reverse rate of the origin of new species, is of the order of several million years (Makarieva and Gorshkov, 2004). Life is over 4 billion years old, while life on land is nearly an order of magnitude younger. It took over 3 billion years for trees to originate in the course of evolution. From the appearance of the first near-water plants in the Ordovician period to the wide spread of ancient forests on land by the late Devonian period, it took nearly a 100 million years to form an ecosystem capable of running a biotic pump and arranging a hydrological cycle on land.

The genetic programs of species that form the ecological community of a natural forest have, therefore, undergone evolutionary optimisation through dozens of millions of years. Information fluxes processed on the molecular level by the living cells of continuous forest cover exceed the information fluxes processed in all the computers of modern civilisation by 20 orders of magnitude - a factor of $10^{20}$ (Gorshkov et al., 2000). This information on the state of the environment is used by the ecological community to initiate controlling processes that keep climates relatively stable and ecosystems in an optimal state for life. The biotic pump mechanism is based on a great variety of complex properties, from the ability of tree roots to spread to great depth and get access to the deeply stored groundwater to the ability of leaves to emit various substances that serve as condensation nuclei and control the condensation process in the atmosphere.

Only the natural ecological community possesses the full set of needed properties to effectively control the water cycle on a given territory. Humanly designed development projects, which introduce alien species that might appear, have 'better' growth, transpiration, or interception, represent a disturbance with respect to natural ecosystem functioning. Such a disturbance can weaken or even totally destroy biotic control of the hydrological cycle and simply planting trees is an ineffectual measure for restoring the complex climate integrative functions of land, plants, water, oceans and moving air. 


\section{Acknowledgements}

We thank two anonymous reviewers for constructive and stimulating criticisms. An anonymous reader of our website is thanked for bringing the heat pipe concept to our attention.

\section{References}

Beresford-Jones, D., Arce Torres, S., Whaley, O. and Chepstow-Lusty, A. (2009) 'The role of prosopis in ecological and landscape change in the samaca basin, lower inca valley, south coast peru from the early horizon to the late intermediate period', Latin American Antiquity, Vol. 20, No. 2, pp.303-332.

Bonan, G.B. (2008) 'Forests and climate change: forcing feedbacks and the climate benefits of forests', Science, Vol. 320, No. 5882, pp.1444-1449.

Chikoore, H. and Jury, M.R. (2010) 'Intraseasonal variability of satellite-derived rainfall and vegetation over Southern Africa', Earth Interactions, Vol. 14, No. 3, pp.1-26.

Duan, J., Bevis, M., Fang., P., Bock, Y., Chiswell, S., Businger, S., Rocken, C., Solheim, F., van Hove, T., Ware, R., McClusky, S., Herring, T.A. and King, R.V. (1996) 'GPS meteorology: direct estimation of the absolute value of precipitable water', Journal of Applied Meteorology, Vol. 35, No. 6, pp.830-838.

Gill, A.E. (1982) Atmosphere-Ocean Dynamics, Vol. 1, Academic Press, New York.

Grover, G.M., Cotter, T.P. and Erickson, G.F. (1964) 'Structures of very high thermal conductance', Journal of Applied Physics, Vol. 35, No. 6, pp.1990-1991.

Gorshkov, V.G., Gorshkov, V.V. and Makarieva, A.M. (2000) Biotic Regulation of the Environment: Key Issue of Global Change, Springer-Praxis, London.

Holt-Giménez, E. and Kenfield, I. (2008) When Renewable Isn't Sustainable: Agrofuels and the Inconvenient Truths Behind the 2007 U.S. Energy Independence and Security Act, Institute for Food and Development Policy, Oakland.

L'vovitch, M.I. (1979) World Water Resources and their Future, American Geological Union, Washington.

Makarieva, A.M. and Gorshkov, V.G. (2004) 'On the dependence of speciation rates on species abundance and characteristic population size', Journal of Biosciences, Vol. 29, No. 1, pp.119-128.

Makarieva, A.M. and Gorshkov, V.G. (2007) 'Biotic pump of atmospheric moisture as driver of the hydrological cycle on land', Hydrology and Earth System Sciences, Vol. 11, No. 2, pp.1013-1033.

Makarieva, A.M. and Gorshkov, V.G. (2009a) 'Condensation-induced dynamic gas fluxes in a mixture of condensable and non-condensable gases', Physics Letters A, Vol. 373, No. 32, pp.2801-2804.

Makarieva, A.M. and Gorshkov, V.G. (2009b) 'Condensation-induced kinematics and dynamics of cyclones, hurricanes and tornadoes’, Physics Letters A, Vol. 373, No. 46, pp.4201-4205.

Makarieva, A.M., Gorshkov, V.G. and Li, B-L. (2008) 'Energy budget of the biosphere and civilization: Rethinking environmental security of global renewable and non-renewable resources', Ecological Complexity, Vol. 5, No. 4, pp.281-288.

Makarieva, A.M., Gorshkov, V.G. and Li, B-L. (2009) 'Precipitation on land versus distance from the ocean: Evidence for a forest pump of atmospheric moisture', Ecological Complexity, Vol. 6, No. 3, pp.302-307. 
Makarieva, A.M., Gorshkov, V.G., Sheil, D., Nobre, A.D. and Li, B-L. (2010) 'Where do winds come from? A new theory on how water vapor condensation influences atmospheric pressure and dynamics', Atmospheric Chemistry and Physics Discussions, Vol. 10, No. 10, pp.24015-24052.

McAlpine, C.A., Syktus, J., Ryan, J.G., Deo, R.C., McKeon, G.M., McGowan, H.A. and Phinn, S.R. (2009) 'A continent under stress: Interactions, feedbacks and risks associated with impact of modified land cover on Australia's climate', Global Change Biology, Vol. 15, No. 9 , pp.2206-2223.

McEwan, M.J. and Phillips, L.F. (1975) Chemistry of the Atmosphere, John Wiley \& Sons, New York.

Mokhin, I.L. and Schlesinger, M.E. (1994) 'Analysis of global cloudiness 2. Comparison of ground-based and satellite-based cloud climatologies', Journal of Geophysical Research, Vol. 99, No. D8, pp.17045-17065.

Montgomery, M.T., Bell, M.M., Aberson, S.D. and Black, M.L. (2006) 'Hurricane Isabel (2003): New insights into the physics of intense storms. Part I: Mean vortex structure and maximum intensity estimates', Bulletin of the American Meteorological Society, Vol. 87, pp.1225-1347.

National Research Council (2005) Radiative Forcing of Climate Change: Expanding the Concept and Addressing Uncertainties, National Academic Press, Washington, p. 92.

Pielke Sr., R.A. (2001) 'Influence of the spatial distribution of vegetation and soils on the prediction of cumulus convective rainfall', Reviews of Geophysics, Vol. 39, No. 2, pp.151-177.

Rex, D.F. (1958) 'Vertical atmospheric motions in the equatorial Central Pacific', Geophysica, Vol. 6, No. 3, pp.479-500.

Schneider, T. (2006) 'The general circulation of the atmosphere', Annual Review of Earth and Planetary Science, Vol. 34, pp.655-688.

Sheil, D. and Murdiyarso, D. (2009) 'How forests attract their rain: an examination of a new hypothesis', BioScience, Vol. 59, No. 4, pp.341-347.

Wood, R., Bretherton, C.S. and Hartmann, D.L. (2002) 'Diurnal cycle of liquid water path over the subtropical and tropical oceans', Geophysical Research Letters, Vol. 29, No. 23, p.GL015371.

\section{Appendix: Condensation in the adiabatically ascending air}

A full system of equations that allows one to determine all variables for moist adiabatic ascent in hydrostatic equilibrium after setting the boundary conditions $T_{s}=T(0), \gamma_{s}=\chi(0)$ at the surface, are as follows (Makarieva et al., 2010, see also equations (1), (3), (13) and Figure 4):

$$
\begin{aligned}
& -\frac{1}{T} \frac{\partial T}{\partial z} \equiv \frac{\Gamma(z)}{T}=\frac{\mu}{h} \frac{1+\gamma \xi}{1+\mu \xi^{2}}, \quad-\frac{1}{\gamma} \frac{\partial \gamma}{\partial z}=\frac{1}{h_{\gamma}}, \\
& h_{\gamma}^{-1} \equiv h_{v}^{-1}-h^{-1}, \quad h_{v}^{-1}=\xi \frac{\Gamma(z)}{T}, \quad h^{-1}=\frac{M g}{R T}, \quad \xi \equiv \frac{L_{v}}{R T}, \quad \mu \equiv \frac{R}{c_{p}} .
\end{aligned}
$$

Heights $h_{v}$ and $h$ are determined by Clausius-Clapeyron law and hydrostatic equilibrium of moist air, respectively; $M$ is molar mass of moist air that depends on $\gamma ; L_{v}, c_{p}$ and $R$ are molar heat of vaporisation of water, molar heat capacity at constant pressure and molar universal gas constant, respectively. 
Figure 4 Dependence on height $z$ of the major distribution functions of moist air for several different values of surface temperature $T_{s}$ satisfying the system of equations (A1) and (A2)
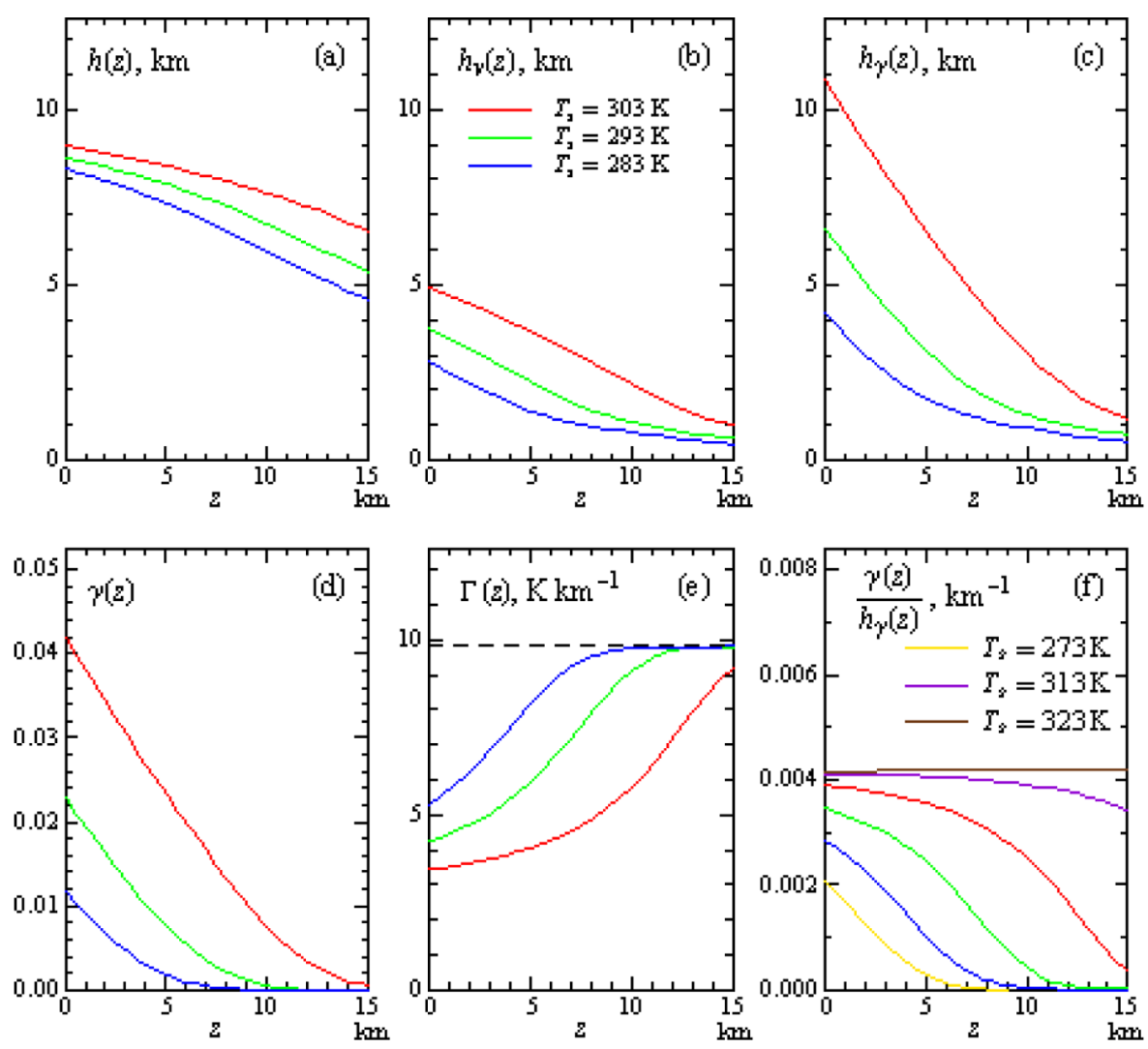

As a separate point, we will now show that equation (14) holds everywhere in the acceptor region. At distance $x$ from the ocean the number of streamlines piercing a vertical cross-section is equal to the number of streamlines leaving the region vertically at the distance from $x$ to $L$. Neglecting the condensation-related loss of matter $(\gamma<<1)$ and cancelling the common multiplier $N$ this condition can be written as

$$
\int_{0}^{h_{\gamma} \alpha(\varsigma)} u(x, z) \mathrm{d} z=\int_{x}^{L} \bar{w}(x, z) \mathrm{d} x .
$$

Here the dimensionless function $\alpha(\zeta), \zeta \equiv(L-x) / L$, accounts for the decrease of the number of streamlines going through a vertical cross-section at point $x$, Figure $2, \bar{w}$ is the mean vertical velocity. At constant $u$ (no horizontal acceleration) and $\bar{w}$ (constancy of precipitation) we have from (A3):

$$
u h_{\gamma} \alpha(\zeta)=(L-x) \bar{w}
$$


This equation has a single solution

$$
\alpha(\zeta)=\zeta=\frac{L-x}{L}
$$

which means that equation (A3) is equivalent to equation (14) at any $x$.

Condensation is accompanied by two processes: a pressure fall and re-distribution of the resulting pressure gradient (relaxation) over the area affected by condensation. Similar processes (pressure rise and relaxation) occur during evaporation. Scale velocity $v_{R}$ characterising the relaxation process is proportional to the relative change of pressure, with the proportionality coefficient describing the mean velocity of thermal motion of molecules (sound velocity $c$ ), $v_{R} \sim(\Delta p / p) c \sim \gamma c \sim 6 \mathrm{~m} \mathrm{~s}^{-1}$. Condensation rate is determined by the vertical velocity $w$ of ascending air, $w \sim 10^{-3} \mathrm{~m} \mathrm{~s}^{-1}$. Therefore, due to $w<<v_{R}$, all the pressure fall caused by condensation appears to be uniformly distributed along the atmospheric column. This accounts for the observed hydrostatic equilibrium.

In local evaporation-condensation eddies with height approximately equal to length we have $u \sim w$ and nearly uniform air pressure along the horizontal part of the eddy. Pressure fall due to condensation is locally compensated by the pressure rise due to evaporation. As the horizontal dimension of the eddy grows, the horizontal velocity $u$ grows as well according to the continuity equation (14). In the result, the horizontal pressure gradient increases to be ultimately described by equation (15) at $u \sim v_{R}$.

Finally, let us find the dependence of $\gamma(x)$ on $x$. The continuity equations (17), (14) together with the dependence of $\gamma$ on $z$ equation (A1) can be combined to yield

$$
\frac{1}{\gamma^{2}} \frac{\partial \gamma}{\partial x}=\frac{1}{L} ; \quad \gamma(x)=\frac{\gamma(0)}{1-\gamma(0) \frac{x}{L}}
$$

That is, $\chi(x)$ equation (A6) grows with $x$, which means that there is evaporation in the acceptor region. At $x=L$ in the innermost point of the acceptor region we have from equation (A6):

$$
\gamma(L)=\frac{\gamma(0)}{1-\gamma(0)}, \quad \frac{p_{v}(L)}{p(L)}=\frac{p_{v}(0)}{p_{d}(0)}, \quad p_{d}(0)=p(0)-p_{v}(0)
$$

Equation (A7) can be interpreted such that all water vapour that has arrived to the acceptor region from the donor region has been spent as the air flow reaches the innermost point $x=L$ of the acceptor region. In such a case the vapour that does remain in the air is the vapour that has evaporated locally in the acceptor region. At $p_{v}(x)=p_{v}(0)$ we conclude that equation (A6) is equivalent to equation (16). If evaporation in the acceptor region tends to zero, i.e., $p_{v}(x) \rightarrow 0$, then $\chi(x) \rightarrow 0$ at any $z$. This means that there cannot be any adiabatic ascent, condensation or precipitation, in short, the acceptor region ceases to exist. 